\title{
Guidelines to develop interactive tutorials on 3D biomolecules: the case of DNA repair by photolyase
}

\author{
Larissa Assis Barony Valadares Fonseca1 ${ }^{1}$ Nadja Cristhina de Souza Pinto ${ }^{1}$, Guilherme Andrade Marson ${ }^{2}$ \\ 1 Universidade de São Paulo, Departamento de Bioquímica (São Paulo, Brasil), 2 Universidade de São Paulo, \\ Departamento de Química (São Paulo, Brasil)
}

Supported by: CAPES

INTRODUCTION: This work presents the following guidelines for developing interactive learning objects on the 3D biomolecules: i- identify a relevant educational need; ii-select an appropriate subject; iii- employ interactive 3D molecular structures; iv- simultaneously display animation with related textual information or 2D diagrams; vintegrating different modes of representation of chemical phenomena. Based on these, was constructed the "Photolyase Tutorial". Photolyase is an enzyme that repairs DNA. It recognizes the cyclobutane pyrimidine dimer lesion (CPD) caused by exposure to ultraviolet light. Understanding the repair mechanism requires understanding the structure-activity relationships of the molecules involved. Therefore, this topic is a convenient subject for teaching key aspects of the structure of protein and nucleic acids. There are few interactive 3D DNA repair tutorials available and most of these are in english, therefore not acessible for students that do not domain this language. Besides, these tutorials generally focus only on displaying structural features without make foster correlations between the structure and chemical aspects of repair mechanisms. OBJECTIVES: In order to construct a interactive tutorial that fills these gaps, the 3D structures of a DNA molecule, a DNA with CPD lesion and the photolyase enzyme were manipulated focusing on structural and chemical explanation. MATERIALS AND METHODS: These structures were obtained from PDB (http://www.rcsb.org/pdb/home/home.do) and manipulated to show biochemical and chemical concepts by using the resources from the LABIQ Platform (http://labiq.is.usp.br). DISCUSSION AND RESULTS: Several concepts are covered, i.e., H-bonding, active site, co-factors etc. The tutorial is organized in small conceptual blocks presenting, in a stepwise fashion, how the enzyme recognizes the DNA lesion and proceeds to repair. Each block comprises a trigger button that controls the interactive molecular viewer from the short text information or 2D diagram. CONCLUSION: This way, we developed a interactive tutorial that combines different modes of representation of chemical and structural concepts of biomolecules in a single interface.

Keywords: interactive tutorials; photolyase; 3D biomolecules 